\title{
Avaliando o papel da procrastinação acadêmica e bem-estar subjetivo na predição da satisfação com o programa de pós-graduação
}

\section{Assessing the role of academic procrastination and subjective well-being in predicting satisfaction with the postgraduate program}

\section{Evaluar el papel de la procastinación académica y el bienestar subjetivo en la predicción de la satisfacción con el programa de postgraduación}

\author{
Ana Karla Silva Soares ${ }^{1}$, ORCID 0000-0001-5306-4073 \\ José Alberto Lechuga de Andrade Filho ${ }^{2}$, ORCID 0000-0002-0311-3372 \\ Maria Gabriela Costa Ribeiro ${ }^{3}$, ORCID 0000-0001-6920-9070 \\ Alessandro Teixeira Rezende ${ }^{4}$, ORCID 0000-0002-5381-2155 \\ ${ }^{12}$ Universidade Federal do Mato Grosso do Sul. Brasil \\ ${ }^{34}$ Universidade Federal da Paraíba. Brasil
}

\begin{abstract}
Resumo: O presente estudo objetivou conhecer em que medida a procrastinação acadêmica e o bemestar subjetivo estão relacionadas com satisfação com a pós-graduação. Participaram 263 pósgraduandos, com idade média de 29 anos $(D P=6.48)$, sendo a maioria do sexo masculino $(67.3 \%)$, solteira (60.1\%) e no mestrado (58\%), os quais responderam a Escala de Procrastinação Acadêmica, Escala de Satisfação com o Programa de Pós-Graduação, Escala de Satisfação com a Vida, Escala de Afetos Positivos e Negativos e perguntas demográficas. Os resultados indicaram que a procrastinação acadêmica se correlacionou negativamente com o bem-estar subjetivo e positivamente com a satisfação com a pós-graduação, sendo desta última preditor. Tal modelo testado apresentou indicadores de ajuste adequados $\left[\chi^{2}(4)=12.73, \chi^{2} / \mathrm{gl}=3.18, \mathrm{GFI}=0.98, \mathrm{AGFI}=0.93, \mathrm{CFI}=0.97\right.$, RMSEA $=0.091($ IC90\%=0.038-0.150) e Pclose $=0.09]$. Conclui-se que a procrastinação acadêmica e bem-estar subjetivo são variáveis importantes para compreender a satisfação com a pós-graduação, favorecendo intervenções que proporcionem melhor adaptação ao contexto de pós-graduação.
\end{abstract}

Palavras-chave: satisfação com a vida, procrastinação, bem-estar, pós-graduandos

Abstract: The present study aimed to know the extent to which academic procrastination and subjective well-being are related to satisfaction with post-graduation. Participants were 263 postgraduate students, with a mean age of 29 years $(S D=6.48)$, with the majority of males $(67.3 \%)$, single $(60.1 \%)$ and the master (58\%), who responded to the Academic Procrastination Scale, Scale of Satisfaction with the Postgraduate Program, Life Satisfaction Scale, Positive and Negative Affect Schedule and demographic questions. The results indicated that academic procrastination (-) and subjective well-being $(+)$ predicted satisfaction with post-graduation. This tested model showed adequate fit indicators $\left(\chi^{2} / \mathrm{g} .1=3.18, \mathrm{GFI}=0.98, \mathrm{CFI}=0.97\right.$, RMSEA $=0.048$ and Pclose $\left.=0.09\right)$. It may be concluded that academic procrastination and subjective well-being are important variables to understand the satisfaction with the postgraduate, favoring interventions that provide better adaptation to the postgraduate context.show the validity of the MBPQS to assess the sensitivity of caregivers of children with ASD.

Keywords: life satisfaction, procrastination, well-being, postgraduate students 
Resumen: El presente estudio tiene por objeto conocer en qué medida la procrastinación académica y el bienestar subjetivo están relacionados con la satisfacción con la posgraduación. Los participantes del estudio fueron 263 estudiantes de posgrado, con edad media de 29 años $(D E=6.48)$, siendo la mayoría del sexo masculino $(67.3 \%)$, soltera $(60.1 \%)$ y en el máster (58\%), los cuales respondieron a la Escala de Procrastinación Académica, Escala de Satisfacción con el Programa de Postgrado, Escala de Satisfacción con la Vida, Escala de Afectos Positivos y. Negativos y preguntas demográficas. Los resultados indicaron que la procrastinación académica (-) y el bienestar subjetivo (+) predijeron la satisfacción con el programa de postgrado. Este modelo probado presentó indicadores de ajuste adecuados $\left(\chi^{2} / \mathrm{gl}=3.18\right.$, GFI $=0.98$, AGFI $=$ 0.93, CFI $=0.97$, RMSEA $=0.091$ y Pclose $=0.09$ ). Se concluye que la procrastinación académica y el bienestar subjetivo son variables importantes para comprender la satisfacción con el programa de postgrado, favoreciendo intervenciones que proporcionen una mejor adaptación al contexto de postgrado.

Palabras clave: satisfacción con la vida, procastinación, bienestar, postgraduados

Recebido: 10/08/2018

Aceito: $27 / 12 / 2019$

Como citar este artigo:

Soares, A. K. S., Andrade Filho, J.A.L., Ribeiro, M. G. C., \& Rezende, A. T. (2020). Avaliando o papel da procrastinação acadêmica e bem-estar subjetivo na predição da satisfação com o programa de pósgraduação. Ciencias Psicológicas, 14(1), e-2078. doi: https://doi.org/10.22235/cp.v14i1.2078

Correspondência: Ana Karla Silva Soares - Universidade Federal do Mato Grosso do Sul, Rua Dr. Werneck, 41, ap. 4202, Vila Albuquerque, CEP: 79060-300, Campo Grande/MS, Brasil, e-mail: akssoares@gmail.com. José Alberto Lechuga de Andrade Filho - Universidade Federal do Mato Grosso do Sul, Rua Dr. Werneck, 41, ap. 4202, Vila Albuquerque, CEP: 79060-300, Campo Grande/MS, Brasil, e-mail: lechugadeandrade@gmail.com. Maria Gabriela Costa Ribeiro - Universidade Federal da Paraíba, Rua João Galiza de Andrade, 492. Jardim São Paulo, CEP: 58051-180, João Pessoa/PB, e-mail: mariagabicr@gmail.com. Alessandro Teixeira Rezende - Universidade Federal da Paraíba, Avenida Carneiro da Cunha -1289, Bairro: Torre, João Pessoa/PB, CEP: 58040-243, e-mail: als_tx29@hotmail.com

Após a conclusão do curso acadêmico, muitos universitários optam pela continuação dos estudos em programas de pós-graduação stricto sensu (mestrado e doutorado). Entretanto, observa-se nos últimos anos, uma maior discussão e preocupação com o bem-estar destes estudantes, visto que este período de transição do ensino superior para o mercado de trabalho e a busca por formação complementar envolve diversas mudanças nos âmbitos acadêmicos, emocionais e sociais (Conley, Kirsch, Dickson, \& Bryant, 2014). Neste sentido, além dos fatores externos, elementos intrínsecos podem influenciar a redução do bem-estar subjetivo dos estudantes de pós-graduação e, por sua vez, a satisfação com o programa de pós-graduação escolhido (McCloskey, 2011).

Pesquisas buscaram identificar quais fatores se relacionavam com a satisfação com o programa de pós-graduação. Este fenômeno pode ser definido como a avaliação cognitiva positiva sobre as experiências em torno do programa de pós-graduação (Celik \& Storme, 2017). Todavia, a percepção positiva dos estudantes sobre os cursos stricto sensu são escassas na literatura, sendo encontrado um estudo similar realizado por Lizote, Verdinelli e Lana (2011), os quais avaliaram o nível de satisfação dos alunos dos cursos de pós-graduação lato sensu [compreendem programas de especialização e incluem os cursos designados como MBA (Master Business Administration)] a partir do modelo proposto por Paswan e Young (2002). O modelo 
formado por cinco dimensões (envolvimento com o professor, interesse do aluno, interação professor-aluno, demandas e organização do curso, empregando a modelagem por equações estruturais), demonstrou que o envolvimento do professor e o interesse do aluno são determinantes da satisfação geral para os cursos de pós-graduação latu sensu, fornecendo indicadores sobre o tema.

Apesar disso, fatores psicológicos envoltos na relação estabelecida entre o aluno e seu programa de pós-graduação precisam ser melhor explorados a fim de ampliar a compreensão do tema e viabilizar maiores atividades e tarefas que possibilitem a adaptação e melhoria na vivência por parte dos pós-graduandos nesta etapa acadêmica. Uma das possíveis dimensões nessa relação é a procrastinação acadêmica, a qual foi conceituada como a tendência de retardar as tarefas acadêmicas a ponto de gerar ansiedade em detrimento das mesmas (Rothblum, Solomon, \& Murakami, 1986). Desse modo, delongar atividades acadêmicas pode aumentar o risco de mal-estar psicológico (Beiter et al., 2015), isto é, elevar os níveis de ansiedade e estresse dada a pressão do tempo na conclusão da tarefa (Glick, Millstein, \& Orsillo, 2014).

Nesta perspectiva, estudos demonstraram o impacto negativo que a procrastinação acadêmica fornece no bem-estar subjetivo (Balkis, 2013; Habelrih \& Hicks, 2015). Em outras palavras, os acadêmicos mais procrastinadores tendem a se apresentar com menor indicador de bem-estar subjetivo e menor satisfação com a vida acadêmica (Balkis \& Duru, 2016; Grunschel, Schwinger, Steinmayr, \& Fries, 2016). Assim, o bem-estar subjetivo se mostra como componente importante no aspecto da saúde geral (Diener, Oishi, \& Lucas, 2015) sendo, portanto, um importante indicador do nível de adaptação dos estudantes e, consequentemente, da satisfação vivenciada pelos mesmos no contexto da pós-graduação.

Considerando a vida acadêmica de estudantes de pós-graduação no que tange aos construtos bem-estar subjetivo e satisfação com o programa de pós-graduação na presença da procrastinação acadêmica, identifica-se uma provável correlação entre os mesmos, tal como observado no contexto da graduação (Balkis \& Duru, 2016). Assim, compreender variáveis relacionadas a satisfação com o programa e o bem-estar subjetivo, a exemplo da procrastinação acadêmica, é uma ferramenta fundamental de promoção de saúde, e consequentemente, redução de transtornos ou doenças proporcionando melhor adaptação ao contexto de pós-graduação.

Portanto, o presente estudo tem por objetivo geral avaliar o papel explicativo da procrastinação acadêmica e do bem-estar subjetivo na satisfação de estudantes com o programa de pós-graduação. Para tanto, inicialmente versa-se sobre a procrastinação acadêmica e o bemestar subjetivo, considerando suas respectivas definições e correlatos. Seguido do panorama de estudos sobre a relação entre satisfação com o programa de pós-graduação, procrastinação e bem-estar subjetivo.

\section{Procrastinação acadêmica}

A procrastinação é uma prática comum (Yockey, 2016), definida como o ato de adiar a realização de atividades de forma deliberada ou não (Kim, Fernandez, \& Terrier, 2017). Pesquisas tem se dedicado a estudar as diferentes conotações que a procrastinação pode apresentar, ora centrando-se nos aspectos funcionais (e.g., buscar por desenvolver tarefas da melhor maneira possível; Chu \& Choi, 2005; Schraw, Wadkins, \& Olafson, 2007), ora nos não funcionais (e.g., dificuldade de finalização de tarefas por alto grau de exigencia; Abramowski, 2018; Yang, Asbury, \& Griffiths, 2018). No contexto acadêmico, sua influência se mostra mais acentuada, por exemplo, Balkis e Duru (2016) afirmam que 80-90\% dos estudantes universitários adiam seus deveres acadêmicos, tornando-se um fenômeno crescente onde estudantes classificados como procrastinadores tendem a apresentar desempenho menor quando comparados a acadêmicos "não procrastinadores" (Closson \& Boutilier, 2017). 
Segundo Steel e Klingsieck (2016), a procrastinação acadêmica é aquela restrita às tarefas e atividades relacionadas e/ou relevantes para o aprendizado e o estudo, sendo identificado na literatura termos intercambiáveis, como "procrastinação de estudantes". De acordo com Balkis (2013), a procrastinação acadêmica é dinâmica, relacionando-se a características pessoais, ambientais e comportamentais. Além disso, sua ocorrência tem sido relacionada a grades curriculares muito extensas, à redução do estado de bem-estar, aos altos níveis de estresse, apresentando-se como um preditor negativo da realização acadêmica e do bem-estar subjetivo (McCloskey, 2011; Steel \& Klingsieck, 2016; Yang et al., 2018).

$\mathrm{O}$ ato de procrastinar está relacionado também à realização de atividades de que se gostem menos. Estudantes que se encontram em situações complexas para iniciar uma tarefa custosa preferem deixá-la e se dedicarem a outra, por vezes, menos importante (Steel \& Klingsieck, 2016). Quanto aos consequentes da procrastinação acadêmica, são comumente mencionados a redução na satisfação com o contexto acadêmico e do bem-estar subjetivo (Morris \& Fritz, 2015; Steel \& Klingsieck, 2016).

Especificamente, Balkis e Duru (2017) identificam que a procrastinação acadêmica estava negativamente relacionada a satisfação com a vida acadêmica e que a variável sexo era importante na relação, visto que homens apresentavam maior nível de procrastinação acadêmica e menor grau de satisfação com a vida acadêmica, além de ter sido observado um efeito moderador da variável sexo no relacionamento entre a procrastinação, o desempenho e a satisfação com a vida acadêmica.

Deste modo, considera-se que se o estudante apresentar uma maior satisfação com, por exemplo, os estudos, o curso ou o programa de pós-graduação é mais provável que ele cumpra seus objetivos e tarefas no prazo, aumentando a sensação de bem-estar e, provavelmente, reduzindo $o$ ato de procrastinação acadêmica. Desta maneira, verificar empiricamente os correlatos da procrastinação acadêmica favorece a compreensão e ampliação das possibilidades de intervir neste contexto (Häfner, Oberst, \& Stock, 2014).

\section{Bem-estar subjetivo}

O Bem-estar Subjetivo (BES) é um termo amplo e abrangente, que se refere as diferentes formas de avaliar a vida ou experiências emocionais referindo-se ao grau com que uma pessoa acredita ou sente que sua vida está indo bem (Diener, Seligman, Choi, \& Oishi, 2018). Pesquisas na área abrangem o entendimento de como as pessoas evoluem durante a vida, envolvendo tanto o momento presente quanto períodos mais longos (e.g, semanas, meses, anos) das reações emocionais, tais como seus julgamentos em dimensões afetivas (Diener et al., 2015) e acerca de sua satisfação com a vida, que pode ocorrer a nível global ou em domínios específicos, incluindo o acadêmico (Balkis, 2013).

É importante ressaltar que apesar da confusão na terminologia, bem-estar subjetivo não é equivalente ao conceito de felicidade, em que este último "é um termo solto e com muitos significados e, portanto, é frequentemente evitado na literatura cientifica" (Diener et al., 2017, p. 87). Em contrapartida, o termo bem-estar subjetivo se refere as diferentes formas de evolução das avaliações que os indivíduos realizam sobre suas experiências pessoais na vida, como afetos positivos, afetos negativos e satisfação com a vida (Diener, 2013).

Nesta direção, entende-se bem-estar subjetivo como a avaliação e o julgamento realizado sobre vida de um indivíduo, sendo o mesmo um construto tridimensional, constituído pelo fator cognitivo, mensurado por meio de indicares de satisfação com a vida e afetivo que envolve as reações emocionais frente aos eventos da vida, podendo se munir de afetos positivos e negativos (Diener et al., 2015; Scorsolini-Comin, Fontaine, Barroso, \& Santos, 2016). No estado de bemestar, experiências emocionais positivas, como felicidade e alegria, fazem-se mais frequentes quando comparadas às negativas, como raiva, vergonha, depressão e ansiedade, além de se 
evidenciar a satisfação com a vida como um todo ou com áreas específicas (Scorsolini-Comin et al., 2016), inclusive no contexto acadêmico. Dessa forma, BES não significa ausência de afetos negativos, mas sim, elevado nível de juízo positivo em relação à vida, além de afetos positivos muito presentes.

Pesquisadores tem dedicado atenção para relação entre indicadores de BES e variáveis escolares, a exemplo das pesquisas que identificaram correlação entre níveis mais elevados de BES e maiores níveis de performance acadêmica, saúde mental, satisfação geral com diferentes aspectos da vida (J.A. Dela Coleta, Lopes, \& Dela Coleta, 2012), incluindo satisfação com a vida acadêmica, que se correlacionou negativamente com a dimensão de BES relativa aos afetos negativos (Balkis \& Duru, 2016), visto que aqueles que encontraram prazer e satisfação com suas atividades acadêmicas tenderam a sentir mais indicadores positivos de BES (e.g., afetos positivos, satisfação com a vida) além de melhorar o desempenho (Bailey \& Phillips, 2016). Estes elementos reforçam a hipótese formulada nesta pesquisa de que o BES estará relacionado a satisfação dos estudantes com seus respectivos programas de pós-graduação.

De acordo com Faro (2013), o manejo de situações estressoras, por meio do processo de enfretamento, traduz-se como mediador da percepção de BES. O processo de enfrentamento pode ser compreendido a partir de atitudes regulatórias do comportamento, as quais envolvem a maneira como as pessoas direcionam e mobilizam suas ações. No contexto de programas de pósgraduação, tais comportamentos podem ser facilmente notados, haja vista este ser um ambiente de constante estresse e, ainda assim, carece de estudantes que mantenham produção acadêmica.

\section{Procrastinação, bem-estar subjetivo e satisfação com o programa}

Haja vista o exposto, denota-se coerência com a hipótese de relacionamento do BES com a satisfação com o programa de pós-graduação. Pode-se compreender que tal satisfação está relacionada à própria satisfação com a vida $(\mathrm{SV})$, sendo o componente cognitivo do BES. Dessa forma, é possível pensar que, quando na ocorrência do BES, também há satisfação do sujeito em relação aos seus estudos, ainda que ele esteja exposto a situações estressoras; sua percepção em relação à vida permanece positiva. Elevados níveis de SV possibilitam bons indicadores de saúde mental e física, denotando também um aumento da percepção do BES (Tomomitsu, Perracini \& Neri, 2014). Uma vez ressaltado o nível de BES, compreende-se que os afetos positivos, tais como prazer, alegria e otimismo, são mais identificados pelo indivíduo do que os afetos negativos, como medo, raiva e desesperança.

No contexto da pós-graduação (mestrado e doutorado), o sujeito permanece envolvido com atividades acadêmicas por longos períodos. É possível denotar, então, que a satisfação com a vida e com a própria pós-graduação está intrinsicamente relacionada ao comportamento procrastinatório, uma vez que o estudante tem de cumprir exigências oriundas da instituição, tais como produção de texto, cumprimento de prazos e frequência em aulas. A não obediência às normas, isto é, procrastinar, pode implicar consequências aversivas ao aluno quando a mesma ocasiona a não conclusão das atividades (e.g. perda de bolsa de financiamento, notas baixas e perda da vaga).

Neste mesmo cenário, o BES também sofre influências, uma vez que o adiamento do início ou da conclusão de uma tarefa pode, temporariamente, afastar afetos negativos, como desesperança, raiva e frustração. No entanto, com a aproximação do dia de uma prova ou da entrega de uma atividade, por exemplo, aqueles mesmos afetos têm maiores probabilidades de serem sentidos, fazendo reduzir a avaliação positiva que o estudante realiza de sua vida como um todo e do ambiente onde essa demanda se origina (pós-graduação).

Em suma, embora sejam identificadas pesquisas que avaliam estas temáticas separadamente, uma análise integrada destas dimensões é escassa considerando o contexto da pós-graduação, algo fundamental para fornecer informações benéficas sobre a natureza da 
relação destes construtos. Nesta direção, esta pesquisa objetiva avaliar o papel explicativo da procrastinação acadêmica e do bem-estar subjetivo na satisfação de estudantes com o programa de pós-graduação.

\section{Método}

\section{Participantes}

Participaram 263 estudantes brasileiros de programas de pós-graduação, com idade média de 29 anos (variando de 21 a 59 anos; $D P=6.48$ ), sendo a maioria do sexo masculino $(67.3 \%)$, solteira (60.1\%), sem filhos $(79.5 \%)$, no mestrado (58\%) e que dedicam, aproximadamente, 4 horas por dia para estudar $(D P=3.49)$, excetuando-se o período em que estão em seus programas de pós-graduação. Contou-se com uma amostra por conveniência não probabilística.

\section{Instrumentos}

Os participantes responderam um conjunto de perguntas sobre a caracterização da amostra (sexo, idade, nível de pós-graduação, estado civil, se possui filho e tempo dedicado aos estudos) e as seguintes medidas:

- Escala de Procrastinação Acadêmica (EPA). Esta medida foi elaborada originalmente por McCloskey (2011) e adaptada para o presente estudo, tendo como objetivo à avaliação de hábitos e rotinas do estudante, por meio de respostas a 25 itens (e.g., Testes foram feitos para serem estudados na véspera; eu retardo meus projetos até o último minuto) respondidos em uma escala de cinco pontos: 1 (discordo totalmente) a 5 (concordo totalmente). Os resultados identificados nesta versão adaptada evidenciam evidências de adequação psicométrica quanto a validade (unifatorial) e precisão (alfa de Cronbach $=.84$ ).

- Escala de Satisfação com o Programa de Pós-Graduação (ESCP). Originalmente elaborada para a graduação por Nauta (2007), foi adaptada para esta pesquisa e visa avaliar a satisfação global dos estudantes com o próprio programa de pós-graduação (e.g., gostaria de estar mais feliz com a minha escolha de programa de pós-graduação), sendo constituído por seis itens respondidos em escala de cinco pontos, variando de 1 (discordo totalmente) a 5 (concordo totalmente). Nauta (2007) identificou consistência interna satisfatória (alfa de Cronbach $=.95$ ), sendo o mesmo verificado no presente estudo (alfa de Cronbach $=.87$ ).

- Escala de Satisfação com a Vida (ESV). Elaborado por Diener, Emmons, Larsen e Griffin (1985), adaptada para o contexto brasileiro por Gouveia, Milfont, Fonseca e Coelho (2009), esta medida é composta por cinco itens e têm o intuito de avaliar o julgamento que as pessoas realizam a respeito do quão satisfeitas estão com suas vidas (e.g., as condições da minha vida são excelentes; estou satisfeito(a) com minha vida). A escala de sete pontos varia de 1 (discordo totalmente) a 7 (concordo totalmente). A consistência da medida neste estudo foi adequada (alfa de Cronbach $=.88$ ).

- Escala de Afetos Positivos e Negativos (EAPN-10). Esta medida foi originalmente proposta por Diener e Emmons (1984), adaptado para o contexto brasileiro por Gouveia et al. (2019). Mensura a valência dos afetos, em que o participante deve assinalar as experiências emocionais nos últimos dias. Esta escala é composta por dez adjetivos, sendo cinco positivos (e.g., feliz, alegre, satisfeito) e cinco negativos (e.g., deprimido, preocupado, frustrado). A consistência interna no estudo foi satisfatória (alfa de Cronbach $=.82$ afetos positivos; alfa de Cronbach $=.81$ afetos negativos). Os itens são respondidos em uma escala de sete pontos, com os extremos entre $1=$ nada e 7 = extremamente. 


\section{Procedimento}

Os dados foram coletados por meio de um questionário online divulgado nas redes sociais, seguindo o procedimento bola de neve. No caso, os pesquisadores divulgaram o link da pesquisa entre os seus contatos, em diferentes redes sociais direcionadas a estudantes e programas de pós-graduação (e.g., facebook e twitter) reforçando na página inicial que a pesquisa era destinada a pós-graduandos (mestrado e doutorado) em atividade. Destaca-se que prévia a participação na pesquisa, os participantes se deparavam e receberam uma via de um termo de consentimento livre e esclarecido (TCLE), contendo informações sobre o caráter anônimo e voluntário da participação que obteve aprovação do Comitê de Ética em Pesquisa (CAAE: 79972517.8.0000.0021), sendo possível declinar a qualquer momento sem que isso acarretasse algum ônus para os voluntários. Em média, os participantes demoravam 15 minutos para responder os instrumentos.

\section{Análise de Dados}

Para a tabulação e análise de dados, utilizaram-se os pacotes estatísticos PASW e AMOS (versão 18). O primeiro, foi empregado para calcular estatísticas descritivas (média, desvio padrão e frequência) e estatísticas inferenciais: correlação $r$ de Pearson, para estimar a direção e a força de relação entre as variáveis; regressão múltipla (método stepwise) para conhecer o poder preditivo da procrastinação acadêmica e bem-estar subjetivo na satifação com programa de pósgraduação; bem como a MANOVA, para verificar as diferenças de média nas variáveis em estudo em função do sexo e do nível de pós-graduação. Foram efetuadas análises exploratórias dos dados, para avaliar a ocorrência de casos ausentes (missing) e outliers, não sendo identificado nenhuma das situações. Para avaliar a normalidade dos dados foi realizado o teste de Normalidade de Kolmorogov-Smirnov, cujos resultados (Ver Tabela 1) identificaram ausência de normalidade na maioria das variáveis (exceto, afetos positivos e negativos), mas prevalência de valores satisfatórios nos indicadores de simetria (menor que 3) e curtose (menor que 8-10) (Marôco, 2014). Entretanto, ver virtude da dimensão da amostra (superior a 25-30) e pautado no Teorema do Limite Central, pode-se considerar que a distribuição da média amostral desta pesquisa que apresenta dimensão razoável $(N=263)$ e aproxima-se à distribuição normal (Marôco, 2014).

Tabela 1

Teste de Kolmogorov-Smirnov, skewness, kurtosis e tamanho do efeito da satisfação com o programa, procrastinação acadêmica e bem-estar subjetivo

\begin{tabular}{lcccc}
\hline Variáveis & Kolmorogov-Smirnov & Skewness & Kurtosis & $p$ \\
\hline $\begin{array}{l}\text { Satisfação com o } \\
\text { programa de } \\
\text { pósgraduação }\end{array}$ & 0.11 & 0.579 & 0.292 & $.001^{*}$ \\
\hline $\begin{array}{l}\text { Procrastinação } \\
\text { acadêmica }\end{array}$ & 0.04 & 0.117 & 0.603 & $.054^{*}$ \\
\hline $\begin{array}{l}\text { Bem-estar subjetivo } \\
\text { Afetos positivos }\end{array}$ & 0.40 & -0.149 & 11.08 & $.001^{*}$ \\
Afetos negativos & 0.06 & -0.015 & -0.281 & .186 \\
Satisfação com a vida & 0.05 & 0.052 & -0.384 & .221 \\
\hline
\end{tabular}

\footnotetext{
$* p<.05$
} 
Por sua vez, o segundo foi utilizado para testagem do modelo explicativo (path analysis). Na ocasião, considerou-se a matriz de covariância, adotando o estimador Maximum Likelihood (ML) e os seguintes indicadores: $\chi^{2} /$ g.l $(2 \geq 5)$; GFI, AGFI, CFI $(\geq 0.90)$ e RMSEA $(\geq 0.10)$ (Hair, Black, Babin, Anderson, \& Tatham, 2015).

\section{Resultados}

Inicialmente, realizou-se uma análise de correlação $r$ de Pearson, objetivando conhecer o padrão de correlações entre a procrastinação acadêmica, o bem-estar subjetivo e a satisfação com o programa de pós-graduação. Os resultados são apresentados na Tabela 2, a seguir.

Tabela 2

Correlação entre satisfação com o programa de pós-graduação, procrastinação acadêmica e bem-estar subjetivo

\begin{tabular}{llllllll}
\hline Construtos & $M$ & $D P$ & 1 & 2 & 3 & 4 & 5 \\
\hline 1. Procrastinação acadêmica & 2.80 & 0.76 & & & & & \\
2. Bem-estar subjetivo & 4.29 & 1.18 & $-0.38^{* *}$ & & & & \\
3. Afetos positivos & 4.17 & 1.12 & $-0.23^{* *}$ & $0.91^{* *}$ & & & \\
4. Afetos negativos & 3.81 & 1.20 & $0.31^{* *}$ & $-0.88^{* *}$ & $-0.63^{* *}$ & & \\
5. Satisfação com a vida & 4.32 & 1.31 & -0.30 & $0.89^{* *}$ & $0.60^{* *}$ & $-0.49^{* *}$ & \\
6. Satisfação com a pós-graduação & 3.74 & 0.91 & $-0.34^{* *}$ & $0.62^{* *}$ & $0.49^{* *}$ & $-0.59^{* *}$ & $0.36^{* *}$ \\
\hline
\end{tabular}

Nota: $* p<.05 . * * p<.001$ (teste bi-caudal). Bem-estar subjetivo representado pelas variáveis 2,3 e 4 .

Observa-se que a procrastinação acadêmica se correlacionou mais forte e significativamente, de maneira negativa, com a satisfação com o programa de pós-graduação ( $r=$ -.34; $p<.001)$, seguida pela satisfação com a vida $(r=-.30 ; p<.001)$ e os afetos positivos $(r=-$ $.23 ; p<.001)$. Ademais, verificou-se que apenas os afetos negativos apresentaram correlação positiva $(r=.31 ; p<.001)$ com a procrastinação acadêmica.

Posteriormente, decidiu-se testar em que medida a satisfação com o programa de pósgraduação é predito pela procrastinação acadêmica e pelo bem-estar subjetivo (pontuação total e por fatores). Nesta direção, procedeu-se uma análise de regressão múltipla, considerando a procrastinação acadêmica e os fatores do bem-estar subjetivo (afetos positivos, negativos e satisfação com a vida) como variáveis preditoras da satisfação com o programa de pósgraduação, adotando o método de estimação stepwise, visto que o mesmo minimiza o efeito de multicolinearidade, seleciona objetivamente as variáveis potencializando o poder preditivo e sendo mais parcimonioso na seleção do modelo adequando-se a natureza exploratória do estudo (Hair, et al., 2015). Os resultados são apresentados na Tabela 3 a seguir.

Tabela 3

Regressão Linear da satisfação com o programa de pós-graduação (procratinaçao acadêmica e bemestar subjetivo como preditores)

\begin{tabular}{lcccccc}
\hline & $\mathbf{R}$ & $\mathbf{R}^{2}$ ajustado & $\mathbf{F}$ & $\mathbf{B}$ & Beta & $\boldsymbol{t}$ \\
\hline Afetos negativos & 0.58 & 0.33 & $\mathrm{~F}(261)=130.75$ & -0.29 & -0.40 & $-6.50^{*}$ \\
Afetos positivos & 0.62 & 0.36 & $\mathrm{~F}(260)=14.75$ & 0.18 & 0.22 & $3.66^{*}$ \\
Procrastinação & 0.63 & 0.39 & $\mathrm{~F}(259)=11.98$ & -0.21 & -0.18 & $-3.46^{*}$ \\
\hline
\end{tabular}

Notas: $* p<.001 ; * * p<.05$. 
Como observado, a satisfação com o programa de pós-graduação, ou seja, a satisfação global dos acadêmicos com o próprio programa, foi explicada satisfatoriamente tanto pela procrastinação acadêmica $(\beta=-0.21, p<.001)$, quanto pelos fatores de afetos positivos $(\beta=$ $0.22, p<.001)$ e negativos $(\beta=-.40, p<.001)$ do bem-estar subjetivo, explicando conjuntamente $39 \%$ da variância ( $\mathrm{R}^{2}$ ajustado). Identifica-se ausência de multicolinearidade no modelo com valores próximos a $1\left(\mathrm{VIF}_{\text {afetos positivos }}=1.68 ; \mathrm{VIF}_{\text {afetos negativos }}=1.77 ; \mathrm{VIF}_{\text {procrastinação }}=1.11\right)$. Entretanto, ressalta-se que satisfação com a vida não explicou diretamente a satisfação com o programa de pós-graduação.

Em seguida, considerando que na literatura a procrastinação acadêmica é considerada como um fator de risco para o bem-estar subjetivo (Grunschel et al., 2016, Grunschel \& Schopenhauer, 2015), isto é, o aspecto afetivo diminui os afetos positivos e eleva os afetos negativos enquanto que na dimensão cognitiva identifica-se a redução na satisfação com a vida e, consequentemente, identifica-se a baixa satisfação com os estudos. Diante deste exemplo de achado, optou-se por testar o modelo explicativo no qual a procrastinação acadêmica prediz o bem-estar subjetivo que, por sua vez, prediz a satisfação com o programa de pós-graduação.

Neste caso, submeteu-se o modelo à modelagem de equações estruturais, sendo que os resultados, no geral, apoiaram a adequação deste modelo $\left[\chi^{2}(4)=12.73, p<.01 ; \chi^{2} / \mathrm{gl}=3.18\right.$, GFI $=0.98$, AGFI $=0.93$, CFI $=0.97$ e RMSEA $=0.09$ (IC90\% $=.038-.150)$, Pclose $=0.09]$. Ressalta-se também que todas as saturações (os lambdas) foram estatisticamente diferentes de zero $(\lambda \neq 0 ; \mathrm{z}>1.96, \mathrm{p}<.05)$. O modelo correspondente é apresentado na Figura 1.

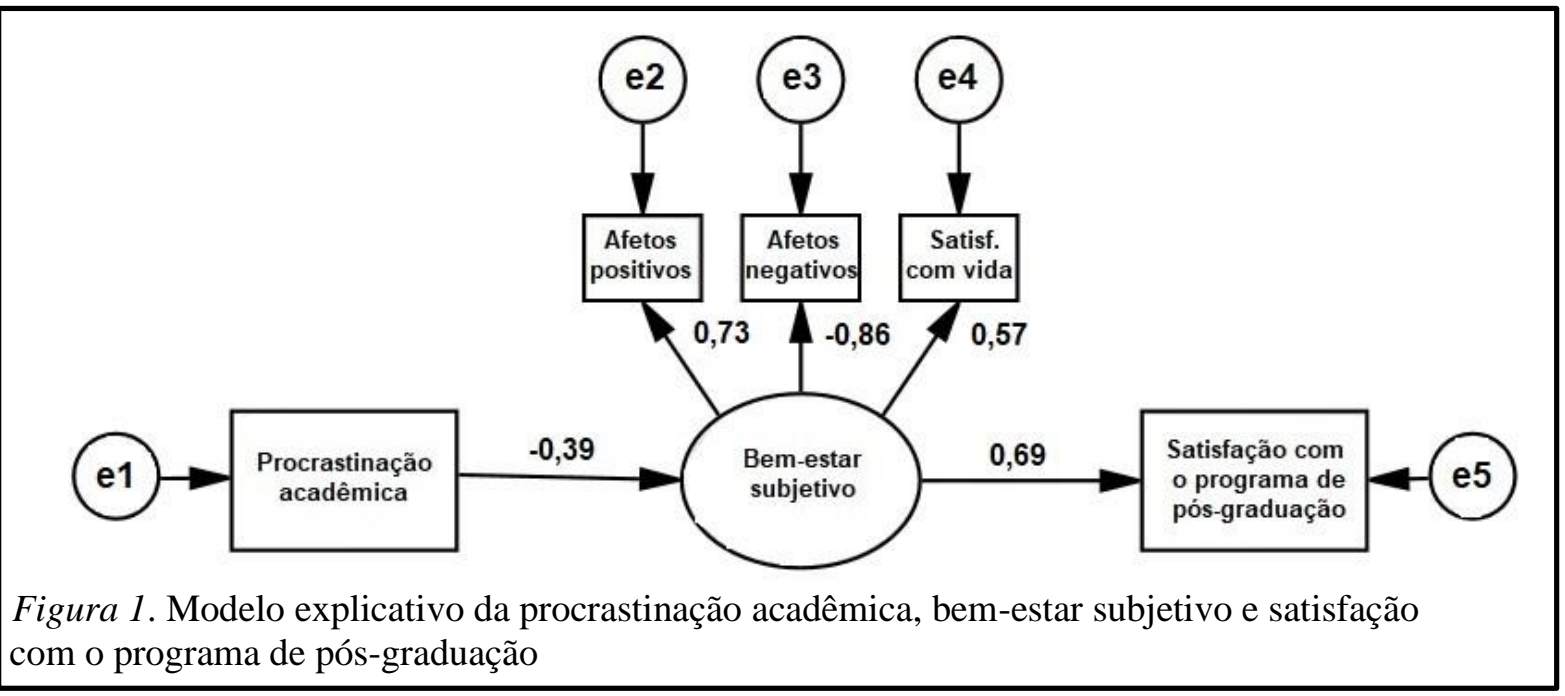

Por fim, visto que há uma diferença entre cursar o mestrado, geralmente a primeira pósgraduação, e o doutorado, bem como avaliar se o sexo pode ser importante para o entendimento do ato de procrastinar, da presença de bem-estar subjetivo e da satisfação com o programa de pós-graduação, optou-se por realizar uma MANOVA tendo os fatores de bem-estar subjetivo, a procrastinação acadêmica e a satisfação com o programa de pós-graduação como variáveis dependentes e o sexo e o nível na pós-graduação (mestrado ou doutorado) como variáveis independentes. Os resultados indicaram que não foram identificadas diferenças estatisticamente significativas nas variáveis sexo [Lambda de Wilks $=0.99, \mathrm{~F}(5,255)=0.64, p>.05, \eta^{2}=0.23$ ] e nível no programa [Lambda de Wilks $\left.=0.99, \mathrm{~F}(5,255)=0.69, p>.05, \eta^{2}=0.25\right]$.

\section{Discussão}

Este estudo teve como objetivo conhecer em que medida a procrastinação acadêmica e o bem-estar subjetivo se relacionam e permitem explicar a satisfação com o programa de pósgraduação. Considera-se que este propósito tenha sido alcançado. Comumente, estudos tem 
demonstrado que a procrastinação acadêmica diminui os indicadores do bem-estar subjetivo (Grunschel et al., 2016) e de forma contrária, aumenta o nível de estresse e ansiedade (Macher, Paechter, Papousek, \& Ruggeri, 2012) na população universitária. Com base nisso, discute-se os principais achados deste estudo que auxilia no preenchimento de possíveis lacunas no que tange a análises das temáticas com estudantes de pós-graduação.

A satisfação acadêmica é compreendida como uma avaliação cognitiva subjetiva acerca das experiências positivas no âmbito universitário (Celik \& Storme, 2017). De forma similar, essa definição pode ser estendida para a satisfação com o programa de pós-graduação, uma vez que a opção do curso ocorre, em geral, com a identificação e vivências positivas por parte da pessoa com a área (Wiswall \& Zafar, 2014). Os indivíduos que se encontram mais satisfeitos no âmbito acadêmico, demonstram níveis maiores de satisfação com a família, amigos e universidade (Balkis, 2013). Por outro lado, pessoas com baixo nível de satisfação acadêmica apresentam isolamento social, dificuldades com colegas de sala (Wilkins-Yel, Roach, Tracey, \& Yel 2018), além de sintomas de estresse e ansiedade (Beiter et al., 2015). Portanto, os resultados deste estudo foram na direção esperada, indicando o conhecimento de uma variável que se relaciona negativamente com a satisfação com a pós-graduação: a procrastinação acadêmica.

Nesta perspectiva, como o entendimento da procrastinação acadêmica se refere a tendência de adiar atividades e comportamentos relacionados à universidade (McCloskey, 2011), pesquisas verificaram os consequentes desta prática. Comumente, a procrastinação acadêmica envolve a demora a finalização de tarefas, projetos e atividades de modo que a corrida contra o tempo, torna-se danoso para o indivíduo, acarretando níveis elevados de mal-estar psicológico (Beiter et al., 2015; Macher et al., 2012). Desse modo, a procrastinação acadêmica ao reduzir os níveis do bem-estar subjetivo (Habelrih \& Hicks, 2015), afeta outras áreas da vida, como os relacionamentos sociais (McCloskey, 2011), nível de realização acadêmica (Balkis, 2013) e, como demonstrando neste estudo, a satisfação com a pós-graduação.

Os achados da relação entre satisfação com a pós-graduação, as dimensões do bem-estar subjetivo, procrastinação acadêmica foram especificadas. Os três fatores que compõe o bemestar subjetivo explicaram positiva (afetos positivos e satisfação com a vida) e negativamente (afetos negativos) a satisfação com a pós-graduação, enquanto a procrastinação acadêmica o fez inversamente. Embora a satisfação com a vida não tenha explicado diretamente a satisfação com a pós-graduação, decidiu-se manter esse componente no modelo explicativo, uma vez que compõe o BES.

Esses dados sugerem que avaliação satisfatória em relação à vida e a frequência de sentimentos positivos (e.g., felicidade, alegria, otimismo) predizem a satisfação com a pósgraduação. Em contrapartida, pessoas que retardam os prazos das atividades acadêmicas, tendem a apresentar sintomas negativos (e.g., tensão, preocupação, frustração), dado a pressão do tempo para a conclusão das tarefas (Balkis \& Duro, 2016; Faro, 2013).

Em relação ao resultado sobre a diferença entre os sexos feminino e masculino na procrastinação acadêmica, encontrou-se que estes não diferiram em suas médias. A respeito disso, há inconsistência na literatura sobre a diferença entre os sexos na procrastinação acadêmica (Özer, Demir, \& Ferrari, 2009). Pesquisas encontraram que o sexo feminino apresentou maior nível de procrastinação acadêmica (Doyle \& Paludi, 1998; Kutlesa, 1998), ao passo que outros estudos demonstraram a prevalência deste construto no sexo masculino (Balkis \& Duru, 2017; Özer et al., 2009). Entretanto, os achados deste estudo corroboraram com aqueles encontrados por Ferrari (2001) e Watson (2001). Já sobre a diferença entre os níveis de mestrado e o doutorado, verifica-se que esses comportamentos independem do grau acadêmico, sendo esse resultado similar aos achados do estudo realizado por Özer et al. (2009) que também não identificaram diferença quanto ao grau acadêmico (nesta pesquisa classificados como recém ingresso/calouro, dois anos de curso, concluintes e graduados). Portanto, evidencia-se que a 
procrastinação acadêmica é uma prática comum entre os estudantes, indiferente no nível acadêmico, isto é, seja no primeiro período de graduação ou no último ano do doutorado.

Os achados reforçam que a satisfação com o programa de pós-graduação está intimamente relacionada a vivência de bem-estar subjetivo e a maneira como os estudantes (sejam homens, mulheres, mestrando ou doutorandos) exercem suas atividades. Assim, evidencia-se a necessidade de ampliar os olhares frente a elementos não apenas estruturais e relacionais (Lizote, Verdinelli, \& Lana, 2011) dos programas de pós-graduações, mas também incluir uma análise de elementos psicológicos (afetivos, cognitivos e comportamentais) para viabilizar maiores níveis de satisfação dos estudantes para com estes contextos e, consequentemente, melhores resultados no que tange ao desenvolvidos das atividades e o próprio processo de aprendizagem.

\section{Conclusão}

Diante do previamente exposto, identificam-se os primeiros achados no contexto brasileiro do impacto negativo que a procrastinação acadêmica exerce no bem-estar subjetivo e, consequentemente, na satisfação com o programa de pós-graduação. Todavia, tal como todo empreendimento científico, são identificadas algumas limitações, a exemplo do fato de que ao centrar em variáveis individuais, a insatisfação com a pós-graduação não deve ser reduzida a procrastinação acadêmica ou BES; outros possíveis elementos preditores necessitarão ser investigados, abarcando aqueles de ordem pessoal (e.g., habilidades sociais, valore humanos, traços de personalidade) e social (e.g., relacionamento com o orientador, situação financeira outra atividade além da pós-graduação, relacionamento familiares e pares), sendo este um intento cientifico que visa ampliar as variáveis outrora analisadas por outros autores (Lizote et al., 2011) e agregar informações que viabilizem a ampliação dos construtos relacionados a temática, sem que se almeje encerrar as discussões sobre a temática apenas nos achados ora apresentados.

Outra limitação neste estudo foi o delineamento adotado, uma vez que não possibilita afirmações de causa e efeito (Pereira, Sindic, \& Camino, 2013), não permitindo assegurar que as pessoas que procrastinam atividades acadêmicas apresentarão necessariamente baixa redução no bem-estar subjetivo; assim, estudos futuros poderão replicar esses resultados, utilizando o método experimental (Pereira et al., 2013), por exemplo, comparando grupos de pós-graduandos avaliados como procrastinadores e não-procrastinadores e suas pontuações em variáveis que avaliem bem-estar subjetivo, podendo expandir a análise para comparação em virtude da natureza do grau acadêmico (mestrado / doutorado).

Portanto, este estudo indicou como a procrastinação acadêmica e o bem-estar subjetivo se relacionam com a satisfação com o programa de pós-graduação. Deve-se focar, principalmente, em meios que viabilizem o aumento do bem-estar subjetivo e da satisfação com o programa dos estudantes de pós-graduação, uma vez que esse fenômeno possui valor prático na vida das pessoas (Maddux, 2018). Por exemplo, a criação de grupos ou núcleos de recepção dos acadêmicos recém inseridos no contexto da pós-graduação com a finalidade de apresentar de maneira humanizada as demandas acadêmicas e pessoais requeridas neste contexto, visando que a ampliação do conhecimento favoreça a redução de hábitos procrastinadores inerente as atividades até então desconhecidas e, por conseguinte, promovendo bem-estar subjetivo e satisfação com o programa. Indivíduos com maior bem-estar subjetivo e satisfação com o contexto de estudo, apresentam sucesso em diferentes áreas na vida, ou seja, são mais sociais, altruístas e ativos; gostam mais de si e dos outros, apresentam melhor habilidade em resoluções de conflito, maior pensamento criativo e experimentam maior saúde física e longevidade (Diener et al., 2017).

Assim, o entendimento destes construtos se apresentam como fator protetivo, visto que ao se evidenciar aspectos psicológicos específicos como parte do processo de aquisição de 
satisfação para com o programa de pós-graduação, permite-se a ampliação do debate sobre o quanto determinados comportamentos, como o de procrastinar em atividades acadêmicas e vivenciar dimensões afetivas e cognitivas (bem-estar subjetivo), podem influenciar no significado que os acadêmicos atribuem as suas atividades nos seus respectivos programas.

Os achados desta pesquisa permitem ampliar as discussões sobre os critérios geralmente empregados para analisar os aspectos que tornam um programa de pós-graduação mais atrativo para seus acadêmicos, visto que boa parte das avaliações centram-se apenas em aspectos estruturais e técnicos (e.g., estrutura física, qualidade do corpo docente, suporte teórico e técnico), minimizando a importância de aspectos individuais e comportamentais singulares a cada pós-graduando, que se adequadamente manejado (e.g., fornecimento de atendimento e orientação pedagógica e psicológica aos acadêmicos recém ingressos) podem favorecer a adaptação e inserção adequada na rotina acadêmica e, consequentemente, maiores níveis de satisfação com seu programa de pós-graduação.

\section{Referências}

Abramowski, A. (2018). Is procrastination all that "bad"? A qualitative study of academic procrastination and self-worth in postgraduate university students. Journal of Prevention \& Intervention in the Community, 46, 158-170. doi:10.1080/10852352.2016.1198168

Bailey, T. H., \& Phillips, L. J. (2016). The influence of motivation and adaptation on students' subjective well-being, meaning in life and academic performance. Higher education research \& development, 35(2), 201-216. doi: 10.1080/07294360.2015.1087474

Balkis, M. (2013). Academic procrastination, academic life satisfaction and academic achievement: the mediation role of rational beliefs about studying. Journal of Cognitive \& Behavioral Psychotherapies, 13, 57-74.

Balkis, M., \& Duru, E. (2016). Procrastination, self-regulation failure, academic life satisfaction, and affective well-being: underregulation or misregulation form. European Journal of Psychology of Education, 31, 439-459.

Balkis, M., \& Duru, E. (2017). Gender differences in the relationship between academic procrastination, satifaction with academic life and academic performance. Electronic Journal of Research in Education Psychology, 15, 105-125. doi: 10.14204/ejrep.41.16042

Beiter, R., Nash, R., McCrady, M., Rhoades, D., Linscomb, M., Clarahan, M., \& Sammut, S. (2015). The prevalence and correlates of depression, anxiety, and stress in a sample of college students. Journal of Affective Disorders, 173, 90-96. doi:10.1016/j.jad.2014.10.054

Celik, P., \& Storme, M. (2017). Trait Emotional Intelligence Predicts Academic Satisfaction Through Career Adaptability. Journal of Career Assessment, 8, 1-12. doi:10.1177/1069072717723290

Chu, C. A. H., \& Choi, J. N. (2005). Rethinking procrastination: Positive effects of" active" procrastination behavior on attitudes and performance. The Journal of social psychology, 145, 245-264. doi:10.3200/SOCP.145.3.245-264

Closson, L. M., \& Boutilier, R. R. (2017). Perfectionism, academic engagement, and procrastination among undergraduates: The moderating role of honors student status. Learning and Individual Differences, 57, 157-162. doi:10.1016/j.lindif.2017.04.010

Conley, C. S., Kirsch, A. C., Dickson, D. A., \& Bryant, F. B. (2014). Negotiating the transition to college: Developmental trajectories and gender differences in psychological functioning, cognitive-affective strategies, and social well-being. Emerging Adulthood, 2, 195-210. doi:10.1177/2167696814521808

Dela Coleta, J. A., Lopes, J. E. F., \& Dela Coleta, M. F. (2012). Felicidade, bem-estar subjetivo e variáveis sociodemográficas, em grupos de estudantes universitários. Psico-USF, 17(1), 129-139. 
Diener, E. (2013). The remarkable changes in the science of subjective well-being. Perspectives on Psychological Science, 8, 663-666. doi: 10.1177/1745691613507583

Diener, E. D., Emmons, R. A., Larsen, R. J., \& Griffin, S. (1985). The satisfaction with life scale. Journal of Personality Assessment, 49, 71-75. doi:10.1207/s15327752jpa4901_13

Diener, E., \& Emmons, R. A. (1984). The independence of positive and negative affect. Journal of Personality and Social Psychology, 47, 1105-1117.

Diener, E., Heintzelman, S. J., Kushlev, K., Tay, L., Wirtz, D., Lutes, L. D., \& Oishi, S. (2017). Findings all psychologists should know from the new science on subjective wellbeing. Canadian Psychology/Psychologie canadienne, 58(2), 87-104. http://dx.doi.org/10.1037/cap0000063

Diener, E., Oishi, S., \& Lucas, R. E. (2015). National accounts of subjective well-being. American Psychologist, 70, 234-242. doi:10.1037/a0038899

Diener, E., Seligman, M. E. P., Choi, H., \& Oishi, S. (2018). Happiest People Revisited. Perspectives on Psychological Science, 13(2), 176-184. doi: $10.1177 / 1745691617697077$

Doyle J. A. \& Paludi M. A. (1998). Sex and gender: The human experience (4 ${ }^{\text {th }}$ ed.). San Francisco: McGraw- Hill.

Faro, A. (2013). Estresse e estressores na pós-graduação: Estudo com mestrandos e doutorandos no Brasil. Psicologia: Teoria e Pesquisa, 29, 51-60. https://revistaptp.unb.br/index.php/ptp/article/view/717/60

Ferrari, J. R. (2001). Procrastination as self-regulation failure of performance: Effects of cognitive load, self-awareness, and time limits on "working best under pressure". European Journal of Personality, 15, 391-406. doi:10.1002/per.413.abs

Glick, D. M., Millstein, D. J., \& Orsillo, S. M. (2014). A preliminary investigation of the role of psychological inflexibility in academic procrastination. Journal of Contextual Behavioral Science, 3, 81-88. doi:10.1016/j.jcbs.2014.04.002

Gouveia, V. V., Milfont, T. L., Fonseca, P. N., \& Coelho, J. A. P. M. (2009). Life satisfaction in Brazil: Testing the psychometric properties of the satisfaction with life scale (SWLS) in five Brazilian samples. Social Indicators Research, 90, 267-277. doi:10.1007/s11205-0089257-0

Gouveia, V. V., Ribeiro, M. G. C., Loureto, G. D. L., Silva Neta, O. F. D., Gouveia, R. S., Vilar, R., \& Freire, S. E. D. A. (2019). Escala de Afetos Positivos e Negativos (EAPN-10): evidências de sua adequação psicométrica. Temas em Psicologia, 27(1), 189-203. doi:10.9788/TP2019.1-14

Grunschel, C., \& Schopenhauer, L. (2015). Why are students (not)motivated to change academic procrastination? An investigation based on the Transtheoretical Model of change. Journal of College Student Development, 56, 187-200. doi:10.1353/csd.2015.0012.

Grunschel, C., Schwinger, M., Steinmayr, R., \& Fries, S. (2016). Effects of using motivational regulation strategies on students' academic procrastination, academic performance, and well-being. Learning and Individual Differences, 49, 162-170. doi:10.1016/j.lindif.2016.06.008

Habelrih, E. A., \& Hicks, R. E. (2015). Psychological well-being and its relationships with active and passive procrastination. International Journal of Psychological Studies, 7, 25-34. https://epublications.bond.edu.au/fsd_papers/247/

Häfner, A., Oberst, V., \& Stock, A. (2014). Avoiding procrastination through time management: An experimental intervention study. Educational Studies, 40, 352-360. doi:10.1080/03055698.2014.899487

Hair, J. F. J., Black, W. C., Babin, B. J., \& Anderson, R. E. (2015). Multivariate data analysis (7th edition). Upper Saddle River, NJ: Prentice Hall. 
Kim, S., Fernandez, S., \& Terrier, L. (2017). Procrastination, personality traits, and academic performance: When active and passive procrastination tell a different story. Personality and Individual Differences, 108, 154-157. doi:10.1016/j.paid.2016.12.021

Kutlesa, N. (1998). Effect of group counseling with university students who complain of procrastination. Unpublished master's thesis, University of Western Ontario, London, Ontario, Canada.

Lizote, S. A., Verdinelli, M. A., \& Lana, J. (2011). Satisfação dos alunos dos cursos de pósgraduação lato sensu: um estudo através da modelagem em equações estruturais. XI Colóquio Internacional sobre Gestão na América do Sul. II Congresso Internacional IGLU. http://repositorio.ufsc.br/xmlui/handle/123456789/25968

Macher, D., Paechter, M., Papousek, I., \& Ruggeri, K. (2012). Statistics anxiety, trait anxiety, learning behavior, and academic performance. European Journal of Psychology of Education, 27, 483-498.

Maddux, J. (2018). Subjective well-being and life satisfaction: an introducion to conceptions, theories, and measures. In: J. Maddux (Ed.). Subjective well-being and life satisfaction. (pp.3-32). New York: Routledge.

Marôco, J. (2014). Análise de Equações Estruturais: Fundamentos teóricos, software \& aplicações ( $2^{\mathrm{a}}$ ed.). Pero Pinheiro: ReportNumber, Lda.

McCloskey, J. D. (2011). Finally, my thesis on academic procrastination (Master's thesis). Retrieved from ProQuest, UMI Dissertations Publishing.

Morris, P. E., \& Fritz, C. O. (2015). Conscientiousness and procrastination predict academic coursework marks rather than examination performance. Learning and Individual Differences, 39, 193-198. doi:10.1016/j.lindif.2015.03.007

Nauta, M. M. (2007). Assessing college students' satisfaction with their academic majors. Journal of Career Assessment, 15, 446-462. https://doi.org/10.1177/1069072707305762

Özer, B. U., Demir, A., \& Ferrari, J. R. (2009). Exploring academic procrastination among Turkish students: Possible gender differences in prevalence and reasons. The Journal of Social Psychology, 149, 241-257. doi:10.3200/SOCP.149.2.241-257

Paswan, A. K., \& Young, J. A. (2002). Student evaluation of instructor: A nomological investigation using structural equation modeling. Journal of Marketing Education, 24, 193202. doi: $10.1177 / 0273475302238042$

Pereira, C. R., Sindic, D., \& Camino, L. (2013). Metodologia da pesquisa em psicologia social. In L. Camino, A. R. Torres, M. E. Lima, \& M. E. Pereira (Eds.), Psicologia social: temas e teorias (pp. 109-186). Brasília: Technopolitik.

Rothblum, E. D., Solomon, L. J., \& Murakami, J. (1986). Affective, cognitive, and behavioral differences between high and low procrastinators. Journal of Counseling Psychology, 33, 387-394. doi:10.1037/0022-0167.33.4.387

Schraw, G., Wadkins, T., \& Olafson, L. (2007). Doing the things we do: A grounded theory of academic procrastination. Journal of Educational psychology, 99(1), 12 -25. doi:10.1037/0022-0663.99.1.12.

Scorsolini-Comin, F., Fontaine, A. M. G. V., Barroso, S. M., \& Santos, M. A. (2016). Fatores associados ao bem-estar subjetivo em pessoas casadas e solteiras. Estudos de Psicologia, 33, 313-324. doi:10.1590/1982-02752016000200013

Steel, P., \& Klingsieck, K. B. (2016). Academic procrastination: Psychological antecedents revisited. Australian Psychologist, 51, 36-46. doi:10.1111/ap.12173

Tomomitsu, M. R. S. V., Perracini, M. R., \& Neri, A. L. (2014). Fatores associados à satisfação com a vida em idosos cuidadores e não cuidadores. Ciencia \& Saúde coletiva, 19, 34293440. doi:10.1590/1413-81232014198.13952013

Watson, D. C. (2001). Procrastination and the five-factor model: A facet level analysis. Personality and Individual Differences, 30, 149-158. doi:10.1016/S0191-8869(00)00019-2 
Wilkins-Yel, K. G., Roach, C. M., Tracey, T. J., \& Yel, N. (2018). The effects of career adaptability on intended academic persistence: The mediating role of academic satisfaction. Journal of Vocational Behavior, 108, 67-77. doi:10.1016/j.jvb.2018.06.006

Wiswall, M., \& Zafar, B. (2014). Determinants of college major choice: Identification using an information experiment. The Review of Economic Studies, 82, 791-824. doi:10.1093/restud/rdu044

Yang, Z., Asbury, K., \& Griffiths, M. D. (2018). An Exploration of Problematic Smartphone Use among Chinese University Students: Associations with Academic Anxiety, Academic Procrastination, Self-Regulation and Subjective Wellbeing. International Journal of Mental Health and Addiction, 1-19. doi:10.1007/s11469-018-9961-1

Yockey, R. D. (2016). Validation of the short form of the Academic Procrastination Scale. Psychological reports, 118, 171-179. doi:10.1177/0033294115626825

Financiamento: Este estudo foi financiado em parte, pela Coordenação de Aperfeiçoamento de Pessoal de Nível Superior - Brasil (CAPES) - Código Financeiro 001 e pela Universidade Federal de Mato Grosso do Sul (UFMS).

Participação dos autores: a) Planejamento e concepção do trabalho; b) Coleta de dados; c) Análise e interpretação de dados; d) Redação do manuscrito; e) Revisão crítica do manuscrito. A.K.S.S. contribuiu em a, c, d, e; J.A.L.A.F. em a, b, d; M.G.C.R. em d,e; A.T.R. em d, e.

Editora científica responsável: Dra. Cecilia Cracco 